\title{
Singular value decomposition analysis of back projection operator of maximum likelihood expectation maximization PET image reconstruction
}

\author{
Vencel Somai, David Legrady, Gabor Tolnai \\ Institute of Nuclear Techniques, Budapest University of Technology and Economics, Budapest, Hungary
}

Radiol Oncol 2018; 52(3): 337-345.

Received 28 August 2017

Accepted 22 February 2018

Correspondence to: Vencel Somai, Budapest University of Technology and Economics, Müegyetem rkp. 3-9. H-1111 Budapest, Hungary. E-mail: vencel.somai@gmail.com

Disclosure: No potential conflicts of interest were disclosed.

\begin{abstract}
Background. In emission tomography maximum likelihood expectation maximization reconstruction technique has replaced the analytical approaches in several applications. The most important drawback of this iterative method is its linear rate of convergence and the corresponding computational burden. Therefore, simplifications are usually required in the Monte Carlo simulation of the back projection step. In order to overcome these problems, a reconstruction code has been developed with graphical processing unit based Monte Carlo engine which enabled full physical modelling in the back projection.

Materials and methods. Code performance was evaluated with simulations on two geometries. One is a sophisticated scanner geometry which consists of a dodecagon with inscribed circle radius of $8.7 \mathrm{~cm}$, packed on each side with an array of $39 \times 81$ LYSO detector pixels of $1.17 \mathrm{~mm}$ sided squares, similar to a Mediso nanoScan PET/CT scanner. The other, simplified geometry contains a $38,4 \mathrm{~mm}$ long interval as a voxel space, detector pixels are assigned in two parallel sections each containing 81 crystals of a size $1.17 \times 1.17 \mathrm{~mm}$.

Results. We have demonstrated that full Monte Carlo modelling in the back projection step leads to material dependent inhomogeneities in the reconstructed image. The reasons behind this apparently anomalous behaviour was analysed in the simplified system by means of singular value decomposition and explained by different speed of convergence.

Conclusions. To still take advantage of the higher noise stability of the full physical modelling, a new filtering technique is proposed for convergence acceleration. Some theoretical considerations for the practical implementation and for further development are also presented.
\end{abstract}

Key words: PET; maximum likelihood expectation maximization reconstruction; positron range; singular value decomposition; convergence speed; transport Monte Carlo,

\section{Introduction}

In emission tomography maximum likelihood expectation maximization (ML-EM) image reconstruction technique ${ }^{1,2}$ has replaced the analytical approaches (e.g. the widely used filtered back projection) in several applications, since ML-EM offers improvements in spatial resolution and stability due to the more accurate modelling of the system and to the ability of accounting for noise structure. ${ }^{3}$ In exchange ML-EM has only a linear rate of convergence ${ }^{2}$ and its computational cost is still tedious even with the rapidly increasing processing capacity of current computers. Thus a significant part of recent research activities aims at accelerating the algorithm. ${ }^{3-5}$ Another important property partly connected to the low convergence rate is the maximal resolution achievable for a given reconstruc- 
tion method given a certain noise level towards which most of the developments are directed..$^{6-9}$

In order to achieve improvement in both convergence rate and spatial resolution an ML-EM positron emission tomography (PET) reconstruction code has been developed with graphical processing unit (GPU) based Monte Carlo engine. ${ }^{10}$ GPUs parallel threads allow for running the inherently parallel neutral particle Monte Carlo transport simulations approximately hundred times faster than on a comparably priced CPU thus significantly reducing the time required for the reconstruction. As increased computational capacity allows for better physics modelling the main novelty of this code is the ability of full particle transport modelling as accurate as it is worthwhile in hope of improving image quality. ${ }^{11}$

Contrary to expectations such faithful physics modelling in the back projection step of the algorithm causes strong artefacts: modelling positron range leads to tissue dependent inhomogeneity artefacts in the reconstructed image. Furthermore, these inhomogeneities disappear when simplified Monte Carlo simulations are used without. All the differences between the two cases occur in the system matrix (derived from the Monte Carlo simulations) of the back projection step. These differences were analysed with respect to the convergence properties and stability to noise in a smaller test system by means of singular value decomposition (SVD) which is a powerful when analysing rectangular matrices. We found a significant advantage of the matrix belonging to the simplified simulations in terms of both singular values and vectors that characterized the convergence properties and stability of the algorithm. In other words more accurate physical modelling is less efficient in terms of convergence and these differences explained the perceived artefacts. However, after numerous iterations accurate modelling gives better reconstruction for low noise cases. Taking advantage of these results we created an a posteriori filtering matrix applied in each iteration after the back projection step with which we could further amplify these differences for speeding up the convergence, but without spoiling the stability to noise.

This paper is organised as follows: in the first subsection of Materials and methods the details of our reconstruction code and a simplified system are described. Second subsection contains the notations. Third subsection gives a short tutorial for SVD including the closely related Picard condition and the corresponding convergence and noise analysis. Results section is divided into four subsections, which present the perceived artefact in detail, the use of faithful modelling and our newly developed SVD filtering method with comparison to the original algorithm. Finally, possible solutions for the implementation are offered with theoretical considerations to further research. Discussion summarises the impact of the SVD analysis and filter and presents our connecting further research goals.

\section{Materials and methods}

A 3D Monte Carlo based ML-EM image reconstruction code named PANNI (PET Aimed Novel Nuclear Imager) has been developed in the framework of the TeraTomo project. ${ }^{12,13}$

PANNI is a Monte Carlo based image reconstruction software written for GPUs using C and CUDA environments surmising roughly 40000 lines. ${ }^{11}$ The key feature of our software is the possibility of faithful Monte Carlo modelling which accounts for positron range, gamma photon-matter interaction and detector response supported by advanced variance reduction methods. Detectors around the object are positioned on a quasi-cylindrical surface with dodecagon cross section. Detector response is either simulated or a pre-generated tabulated response function may be used. Positron range modelling simplifies to the following probability density function. ${ }^{14}$

$\wp(r)=a A^{2} r e^{-A r}+b B^{2} r e^{-B r}$

with $r$ being the positron range distance, $a, A, b$ and $B$ material dependent constants.

As sampling each of the terms is equivalent to sampling the sum of two exponentially distributed random variables $x$ can be obtained by using double exponential sampling. ${ }^{15}$ Advanced variance reduction methods are implemented for source angular sampling outgoing direction and energy biasing and for free flight sampling. The Monte Carlo engine has been validated against MCNP5. ${ }^{16}$ The code is capable of simulating $10^{8}$ photon pairs per second on a commercially available GPU (NVidia GeForce 690).

Both the forward projection and the back projection steps are carried out via the Monte Carlo method. In the back projection step some of the physics modelling may be turned off.

The code has been tested with two geometries, a sophisticated scanner geometry ("full system") and a simplified smaller system ("1D model"). Acquisition geometry for the full system can be set as wished, in our current setup it consists of a 
dodecagon with inscribed circle radius of $8.7 \mathrm{~cm}$, packed on each side with an array of $39 \times 81$ LYSO detector pixels of $1.17 \mathrm{~mm}$ sided squares comparable to a small animal PET scanner similar to the Mediso nanoScan PET/CT scanner. Coincidence counting is accepted between detector pixels on opposite and next to opposite dodecagon sides (1:3 coincidence). The voxel space of the full system is divided into $128 \times 128 \times 128$ voxels $(0.3 \mathrm{~mm}$ sided $)$ and contains a water-cylinder (light grey area in Figure 1 and Figure 2) except for a smaller cylindrical area containing bone material (dark grey area in Figure 1 and Figure 2) Activity phantom for the evaluation is a cylindrical ring of ${ }^{15} \mathrm{O}$ partially located in bone material (the more commonly used ${ }^{18} \mathrm{~F}$ gives less conspicuous results). From now on simplified modelling means the neglect of the positron range effect in the back projection Monte Carlo simulations in contrast with faithful modelling which accounts for positron range.

The voxel space of the $1 D$ model is a $38.4 \mathrm{~mm}$ long interval containing 256 voxels half of which is located in bone material the other half in water. Detector pixels are assigned in two parallel sections each containing 81 crystals of a size 1.17.x.1.17 mm. Every pixel is in coincidence with every pixel on the opposite side. Roughly speaking the $1 D$ mod$e l$ is a cross-section of the full system geometry of PANNI (Figure 3).

The 1D model contains only positron range modelling, neither gamma photon-matter interaction nor detector response modelling is included. Detection is based on the angle of view of the detector from a given voxel. The two analysed settings are: positron
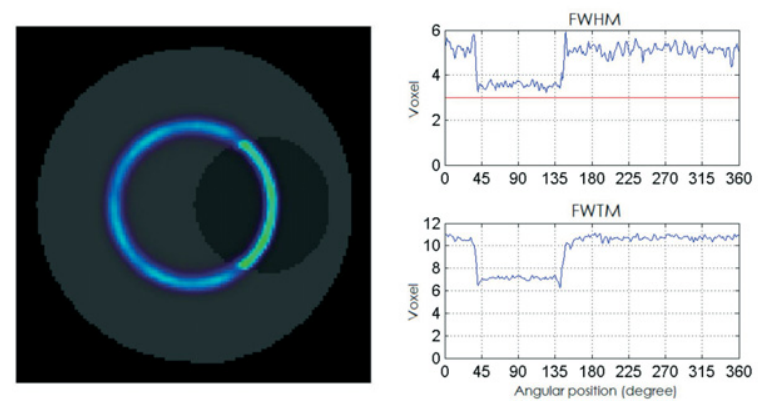

FIGURE 1. Top view of the reconstruction of the cylinder-ring mathematical phantom of the full system with faithful modelling in the back projection. Light grey area represents water, dark grey area represents bone material. Underestimated activity and increased full width at half maximum (FWHM) / full width at tenth maximum (FWTM) can be seen for voxels located in water. FWHM and FWTM are calculated along the ring. Red line indicates the phantom ideal FWHM.
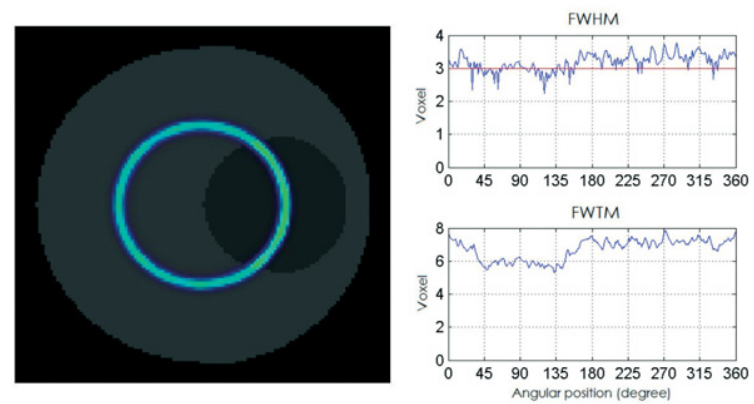

FIGURE 2. Top view of the reconstruction of the cylinder-ring mathematical phantom of the full system with simplified modelling in the back projection. Homogeneous activity estimate and full width at half maximum (FWHM) can be seen along the ring, neglect of positron range in theback projection abolished the artefact of Figure 1 and phantom ideal FWHM is reached.
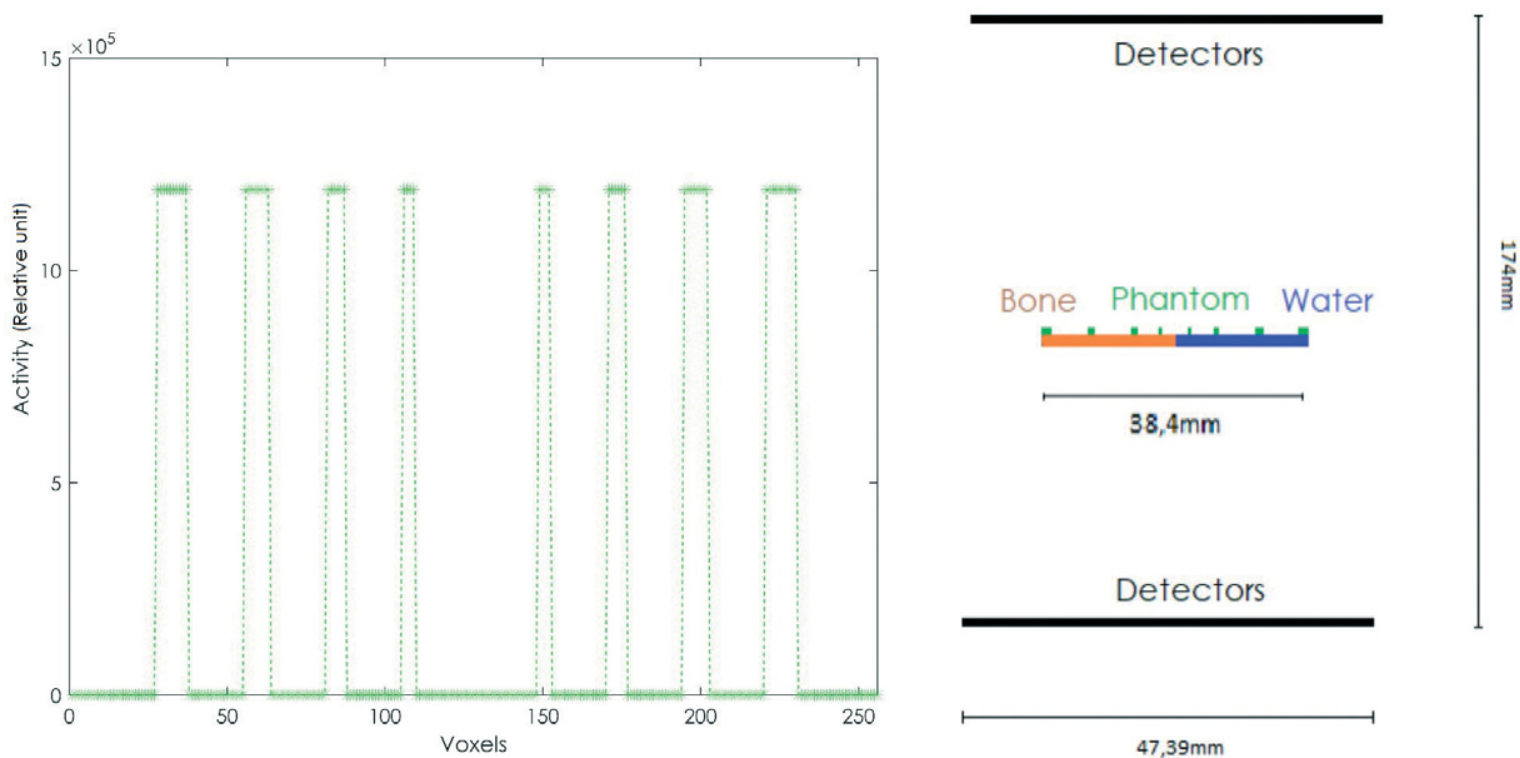

FIGURE 3. Mathematical phantom and system geometry for ID model. 1-128 voxels are located in bone material, 129-256 voxels are located in water. 
range neglected (back projection posrange OFF) and positron range modelled (back projection posrange $\mathrm{ON}$ ) in the back projection. Forward projection always accounts for positron range.

\section{Notations}

$x$ - vector of activity estimate in the voxels, $x \in \mathbb{R}_{\geq 0}^{\text {Nvoxel }}$

$y_{\mathrm{m}}$ - vector of measured data

$y$ - vector of forward-projected data

$y_{\mathrm{r}}$ - pointwise (i.e. Hadamard) ratio vector of measured and forward-projected data $y_{r}=\frac{y_{m}}{y}$

$v_{\mathrm{i}}-\mathrm{i}^{\text {th }}$ singular vector corresponding to voxel space

$\sigma_{\mathrm{i}}-\mathrm{i}^{\text {th }}$ singular value

Lor - Line of response

$u_{\mathrm{i}}-\mathrm{i}^{\text {th }}$ singular vector corresponding to sinogram space

$T$ in superscript means transpose

$A$ - system (response) matrix

$A x$ - forward projection

$A^{\mathrm{T}} y_{\mathrm{r}}$ - back projection

Back projection posrange OFF - simulation ne-

glects positron range in the back projection

Back projection posrange $\mathrm{ON}$ - simulation accounts for positron range in the back projection

FWHM - Full Width at Half Maximum

FWTM - Full Width at Tenth Maximum

$\mathrm{L}_{2}$-norm - Vectorial $\mathrm{L}_{2}$-norm divided by the $\mathrm{L}_{2}$-norm of the activity distribution and multiplied by 100 :

$L_{2}-$ norm $=100 * \sqrt{\frac{\sum_{i=1}^{\text {Nvoxel }}\left(x_{i, \text { phantom }}-x_{i, \text { recon }}\right)^{2}}{\sum_{i=1}^{\text {Nvoxel }} x_{i, \text { phantom }}^{2}}}$

\section{SVD analysis}

The effect of positron range modelling and the average positron range is accounted for by the system matrix. As an obvious tool for the analysis of rectangular matrices, the algorithm and the back projection step is examined by means of singular value decomposition. SVD is a factorisation of any $m \times n$ real or complex matrix $A$ of the form $A=U D V^{\mathrm{T}}$. The following notation is used: $U$ is an $m \times n$ matrix, $D$ and $V$ are $n \times n$ square matrices. In general, matrix $V$ has k orthogonal columns where $\mathrm{k}$ is the rank of the system matrix $A$. $V$ can be completed to a $n \times n$ dimensional matrix by adding $n-k$ orthogonal vectors from the null space of $A^{\mathrm{T}}$ to form a basis in the voxel space. The first $k$ columns of $\boldsymbol{U}$ are also orthogonal and can also be completed to a basis in the sinogram space by adding $m-k$ orthogonal vectors from the null space of $A$. In this last case, $D$ is zero filled to a $m \times n$ dimensional matrix. In the point of view of our analysis the completion of $\boldsymbol{U}$ is not needed and we chose the nomenclature where $\mathrm{U}$ has only $n$ (as $A$ has full rank, thus $k=n$ ) columns. D and $V n \times n$ in this case.

SVD was used for the analysis of convergence speed of the reconstruction algorithm with respect to the applied back projection. According to Liu et al. ${ }^{17}$ the speed of convergence of PET ML-EM algorithm particularly depends on the singular values of the back projection system matrix. Singular values represent relative weights for the voxel space basis vectors (i.e. corresponding voxel space singular vectors) in the update process of the previous activity estimate in a given iteration.

Sinogram space singular vectors can measure the information content of a given measurementforward projection Hadamard ratio in the corresponding back projection step by means of Picard condition formalism. For the existence of a square integrable solution to the problem $y=A x$ the following has to be true ( $A$ is the integral operator the discretization of which is the system matrix $A) .{ }^{18}$

$\sum_{i=1}^{\infty}\left(\frac{u_{i}^{T} y}{\sigma_{i}}\right)^{2}<\infty$

In case of matrices instead of integral operators, the discrete Picard condition requires the spectral coefficients $\left|u_{i}^{T} y\right|$ to decay faster in average than the singular values. ${ }^{18}$ Despite back projection is not a direct inversion from this point of view the faster is the decay of the spectral coefficients $\left|u_{i}^{T} y_{r}\right|$ of the ratio as the index increases the heavier the blurring of the back projection. Higher frequency components level off at a plateau which is dominated by noise and can be regarded as an error-level estimate ${ }^{18}$ because these components do not contain information for the corresponding back projection. Even accounting for voxel space effects only (e.g. positron range) sinogram space singular vectors are not the same for the simplified and faithful modelling. The back projection step of the algorithm back projects the Hadamard ratio of the measured and the currently forward-projected data.

$\boldsymbol{A}^{T} y_{r}=\boldsymbol{V} \boldsymbol{D}^{\boldsymbol{T}} \boldsymbol{U}^{T} y_{r}$

The aforementioned difference in the sinogram space basis affects the $\boldsymbol{U}^{\boldsymbol{T}} y_{r}$ product, i.e. the spectral coefficients of the Hadamard ratio.

\section{Results}

We have compared two reconstruction results for the sophisticated scanner geometry: one with full 
physics modelling in the back projection (Figure 1) and one omitting positron range in the back projection (Figure 2). After 80 iterations faithful modelling produced the reconstruction in Figure 1. The cross section of the cylindrical ring phantom in radial direction is originally a box function which is blurred due to gridding and averaging in a given voxel (similarly to partial volume effect). Therefore, it can be well approximated by a gaussian with a Full Width at Half Maximum of 3 voxels which is indicated by the red line in Figure 1 and Figure 2. The Full Width at Half Maximum/Full Width at Tenth Maximum is calculated by fitting a gaussian in radial direction along the ring separately for each angular position with resolution of 1 degree.

Accounting for positron range in the back projection caused systematic inhomogeneity in the reconstructed image in Figure 1. The activity estimation in the bone material is appropriate (FWHM = 3.5 voxel) in contrast with the activity of the voxels located in water which is underestimated (FWHM $=5$ voxels). The inhomogeneity reduces with simplified back projection, when positron range is neglected in the Monte Carlo simulation. Also the FWHM is reduced in the water area (Figure 2).

The effect of modelling any physical phenomenon appears in the system matrix, thus our analysis aims at finding the differences in the back projection system matrix caused by positron range. Due to its size the system matrix of the full system cannot be stored thus the $1 D$ model was used for further calculations. For real and valid results a comparison of the test system with the full system is needed. Simulations confirmed the analogous behaviour as the perceived artefact appeared in the case of positron range modelling in the back projection while neglecting positron range abolished the problem. Thus, the analysis focuses on the positron range effect, back projection posrange OFF and back projection posrange ON settings are compared. (The positron range free path is sampled with two random variables similarly to the full system). As the corresponding system matrix is of a size 6561x256 it can be directly stored and also the numerical SVD calculation may be carried out.

Comparing the back projection posrange OFF and back projection posrange $\mathrm{ON}$ case in terms of singular values of the system matrix, Figure 4 shows the positive difference for the first 133 index belonging to the former setting.

In the light of the convergence analysis of Ref. ${ }^{17}$ smaller singular values mean that the corresponding frequency components of the solution are later

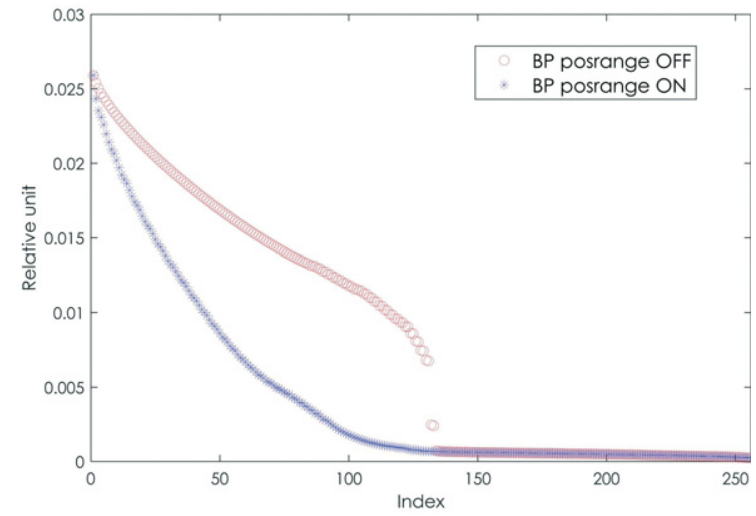

FIGURE 4. Singular values of the system matrix for positron range neglecting and modelling case. Increased values for the former imply the faster convergence of the corresponding (first 133) basis components of the activity estimate.

reconstructed with positron range modelling compared to the positron range neglecting back projection. This space frequency characterises the singular vectors of the voxel space (Figure 5), which is a second but not less significant difference between the two types of system matrices.

Figure 5 on the left accounts only for the symmetries of the system while on the right reflects also the tissue map of the volume. As the average positron free path is much longer in water than in the bone material space frequency of every basis vector is smaller in the area located in the water. Thus the reconstruction of the activity of these voxels is significantly slower and this property is the reason of the obtained artefact resulted from faithful modelling in the back projection and the solution to the perceived anomalous behaviour.

The third and final difference occurs in the sinogram space basis vectors with which the measurement-forward projection Hadamard ratio can be unfolded in a given iteration. The absolute value of the obtained spectral coefficients can be seen in Figure 6 after 15 iterations for the positron range neglecting and modelling case respectively (the spectrum varies slowly through iterations).

Faster decay in the spectral coefficients equals to heavier blurring in the back projection. ${ }^{18}$ This means that the positron range modelling gathers less information from the Hadamard ratio in a given iteration than the positron range neglecting back projection. This also implies the faster convergence and higher stability to noise. 

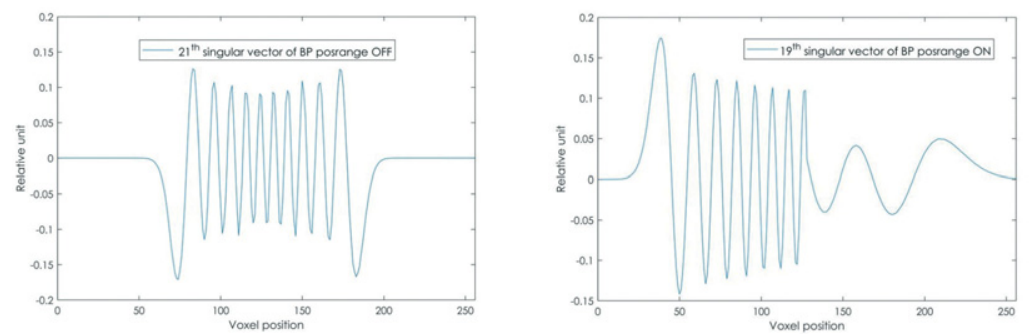

FIGURE 5. One of the voxel space singular vectors of the system matrices corresponding to positron range neglect (left - back projection posrange OFF) and positron range modelling (right - back projection posrange ON). Back projection posrange OFF reflects only the symmetries of the geometry. Back projection posrange $\mathrm{ON}$ accounts for the material map as well, increased position uncertainty due to longer average positron free path implies smaller space-frequency in water area.
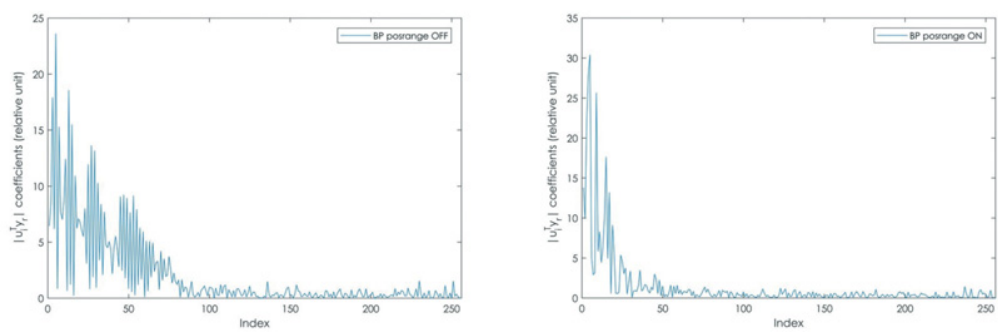

FIGURE 6. Absolute value of the spectral coefficients of the measurement-forward projection Hadamard ratio in the sinogram basis corresponding to positron range neglect (left - back projection posrange OFF) and positron range modelling (right - back projection posrange ON). Faster decay means less information gathered as the coefficients of the horizontal plateau are corrupted by noise thus it represents an error level estimate. Due to one to one correspondence property of SVD between sinogram and voxel space singular (basis) vectors these basis coefficients of the activity are not hoped to be correctly estimated
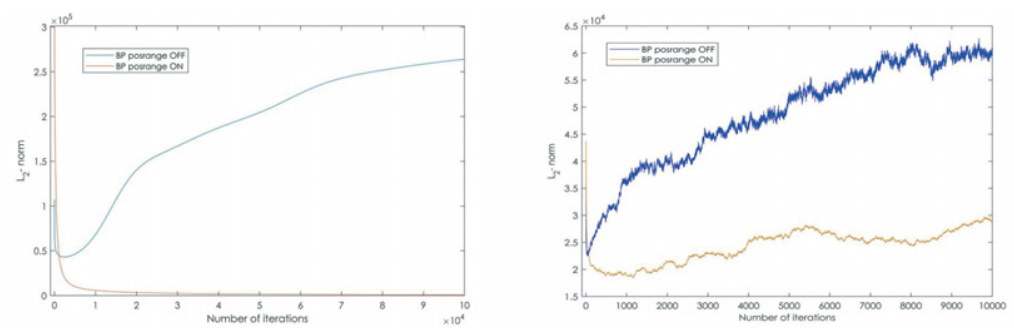

FIGURE 7. The $\mathrm{L}_{2}$-norm of the difference between the activity distribution and the current estimate after a given number of iterations. Smaller value means better agreement. Subfigure on the left shows the result of the noiseless test case where the convergence of back projection posrange ON setting to the exact solution and the convergence of back projection posrange OFf setting to an other fix point is presented. Subfigure on the right shows the result of a simulated reconstruction with $10^{6}$ positron used for measurement generation and in both forward and back projection Monte Carlo simulations. After slower initial convergence back projection posrange ON reaches much better activity estimate. Back projection posrange OFF converges to a different fix point similarly to noiseless case.

\section{Advantage of faithful modelling}

ML-EM with faithful modelling converges to the exact solution. ${ }^{1,2}$ Algorithm using simplified back projection converges to different fix point due to unmatched forward-backward projector pair and only approximates the solution. ${ }^{2,3,5}$ These statements were verified by test simulations with varying noise level (Figure 7). In presence of high noise semi-convergence property of the algorithm ${ }^{3,19}$ is dominant, reaching the optimum estimate as fast as possible is desired which is the advantageous property of the simplified back projection. In low noise case after numerous iterations the faithful modelling outperforms the simplified one reaching much better activity estimate (Figure 7). SVD analysis showed the disadvantage of the former in the term of convergence speed but due to matched forwardbackward projector pair it converges to the exact solution ${ }^{1}$ in contrast with the simplified modelling.

This result resolves the contradiction as additional information indeed leads to better image reconstruction thus the perceived anomaly was only apparent. Simplification just luckily affects the behaviour of the algorithm from a mathematical point of view. However, this form is not the ideal back projection operator but the one that is easy to implement without much modification to the original algorithm. To obtain a better back projection operator the previously listed advantages can be amplified with a posteriori manipulation and a close to ideal form can be reached. As a possible degree of freedom, singular values of the simplified back projection operator can be further increased similarly to the accompanying effect of the simplified modelling. In this case $\boldsymbol{U}$ and $\boldsymbol{V}$ matrices are unchanged. The possible fix points of the algorithm can be obtained from the next equations (ratio is in a Hadamard sense) as the update process multiplies (also in Hadamard sense) the current estimate by 1 when the following is true:

$\frac{\boldsymbol{A}^{\boldsymbol{T}} y_{r}}{|\boldsymbol{A}|}=\frac{\boldsymbol{A}^{\boldsymbol{T}} y_{r}}{\boldsymbol{A}^{\boldsymbol{T}} *\left(\begin{array}{c}1 \\ \vdots \\ 1\end{array}\right)_{\text {Lor-space }}}=\left(\begin{array}{c}1 \\ \vdots \\ 1\end{array}\right)_{\text {voxel space }}$

Rearranging:

$\boldsymbol{A}^{T}\left(y_{r}-\left(\begin{array}{c}1 \\ \vdots \\ 1\end{array}\right)\right)=\boldsymbol{A}^{T} \tilde{y}=0$

Using the dyadic definition of SVD:

$\boldsymbol{A}^{\boldsymbol{T}} \tilde{y}=\left(\sum_{r=1}^{\operatorname{Rank}(\boldsymbol{A})} \sigma_{r} v_{r} u_{r}^{T}\right) * \tilde{y}=0$ 
$v_{r}$ and $u_{r}$ are the columns of matrix $V$ and $U$ respectively. As $V$ is an orthogonal matrix the linear combination above equals to zero precisely when every $\sigma_{r} u_{r}^{T} \tilde{y}$ coefficient equals to zero. The singular values are all nonzero thus the $u_{r}^{T} \tilde{y}$ dot product equals to zero for $\forall r$. This implies that $\boldsymbol{U}^{T} \tilde{y}=0$. Matrix $U$ remains the same with singular value modification so the possible fix points are unchanged.

\section{SVD filter}

The easiest way to modify the singular value spectrum of the back projection system matrix is the application of a matrix of the form:

$B=V D^{*} V^{T}$

$\boldsymbol{D}^{*}$ is the matrix with which $\boldsymbol{D}^{*} \boldsymbol{D}$ has the desired form. In noiseless (test) case, i.e. when there is no noise added to simulations, this form is (the scalar multiple of) the identity matrix, in agreement with the convergence analysis ${ }^{5,17,20}$, as the singular values are clustered together as far as possible. The SVD filter fastens the convergence with two orders of magnitude. However, it cannot be applied straightforward for the real, noisy case.

The measurement process equals to $\boldsymbol{A} x=\boldsymbol{U} \boldsymbol{D} \boldsymbol{V}^{\boldsymbol{T}} x$ where $x$ stands for the activity distribution. Thus, the measurement attenuates its frequency components according to the singular value spectrum (multiplication with matrix $\boldsymbol{D}$ ) and adds some noise to the result. As so only those components which fit to the discrete Picard condition (Figure 6) can be amplified.

We performed several reconstructions with such an SVD filter. The best result was obtained when $\boldsymbol{D}^{*}$ diagonal matrix contains elements of the form (Figure 8):

$\left\{\begin{array}{l}\frac{1}{\sigma_{i}^{p}} \text { if } i<c u t-o f f \\ 0 \text { if } i \geq c u t-o f f\end{array}\right.$ for some $0<p=1-\varepsilon$ and small $\varepsilon$. for some and small.

Then $\boldsymbol{D}^{*} \boldsymbol{D}$ is also diagonal with the following en-

tries: $\begin{cases}\sigma_{i}^{1-p} & \text { if } i<c u t-o f f \\ 0 & \text { if } i \geq c u t-o f f\end{cases}$

which are closer to 1 , so the singular value spectrum of the resulted back projection system matrix is contracted.

The conventional ML-EM formula looks like as follows $\left(y_{\mathrm{r}}\right.$ is the pointwise, i.e. Hadamard ratio vector of measured and forward-projected data, $y_{r}=\frac{y_{m}}{A x} . \mathbf{1}$ is a sinogram space vector containing ones in every coordinate):

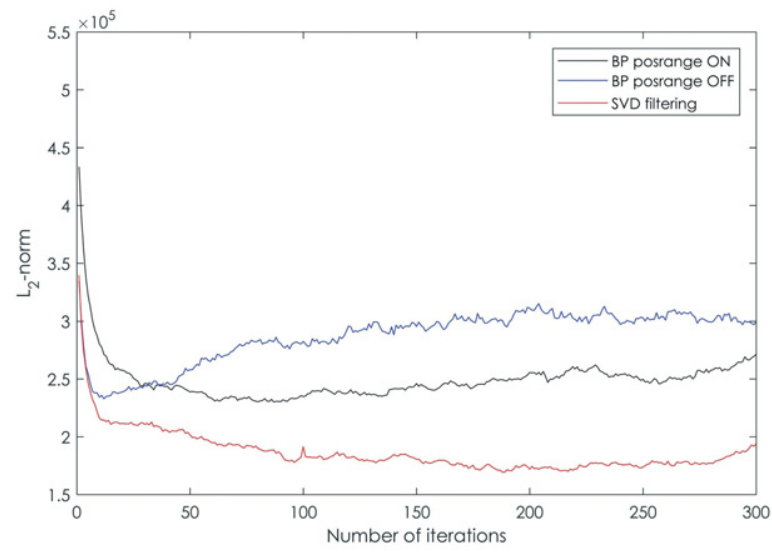

FIGURE 8. The $L_{2}$-norm of the difference between the activity distribution and the current estimate after a given number of iterations. Smaller value means better agreement. Reconstruction with SVD filter outperforms the best setting so far in terms of faster initial convergence and the farther starting point of increasing discrepancy due to semi-convergence. Also the faster initial convergence of positron range neglecting back projection can be seen compared to positron range modelling back projection.

$x^{n+1}=x^{n} \frac{\boldsymbol{A}^{T} y_{r}}{\boldsymbol{A}^{T} \mathbf{1}}=x^{n} \frac{\boldsymbol{V} \boldsymbol{D} \boldsymbol{U}^{T} y_{r}}{\boldsymbol{V} \boldsymbol{D}^{T} \boldsymbol{U}^{T} \mathbf{1}}$

Instead, we use the modified iteration formula which looks like as follows $\left(\boldsymbol{B}^{\boldsymbol{T}}=\boldsymbol{B}\right.$ as being symmetric and $\boldsymbol{V}^{\boldsymbol{T}} \boldsymbol{V}=\boldsymbol{I}$ as $\boldsymbol{V}$ is orthogonal. $\boldsymbol{I}$ is the identity matrix. The ratio and the multiplication in the update process of $x^{n}$ is in Hadamard sense):

$x^{n+1}=x^{n} \frac{\boldsymbol{B} \boldsymbol{A}^{T} y_{r}}{\boldsymbol{A}^{T} \boldsymbol{B} \mathbf{1}}=x^{n} \frac{\boldsymbol{V} \boldsymbol{D}^{*} \boldsymbol{V}^{T} \boldsymbol{V} \boldsymbol{D} \boldsymbol{U}^{T} y_{r}}{\boldsymbol{V} \boldsymbol{D}^{* T} \boldsymbol{V}^{T} \boldsymbol{V} \boldsymbol{D}^{T} \boldsymbol{U}^{T} \mathbf{1}}=x^{n} \frac{\boldsymbol{V} \boldsymbol{D}^{*} \boldsymbol{D} \boldsymbol{U}^{T} y_{r}}{\boldsymbol{V} \boldsymbol{D}^{* T} \boldsymbol{D}^{T} \boldsymbol{U}^{T} \mathbf{1}}$

Figure 8 shows the result of the reconstruction compared to regular ML-EM algorithm with both back projection posrange $\mathrm{ON}$ and back projection posrange OFF settings $\left(6 \times 10^{5}\right.$ positron used). The $\mathrm{L}_{2}$-norm of the difference of the activity distribution and the current activity estimate is presented for each setting after a given number of iterations. Smaller $\mathrm{L}_{2}$-norm means better agreement.

SVD filter outperforms the best setting so far as the initial convergence is faster and better agreement is reached in every iteration. Furthermore, the rise of the discrepancy due to semi-convergence occurs later. Additionally, the faster initial convergence of the positron range neglecting back projection (back projection posrange OFF) can be seen compared to back projection posrange $\mathrm{ON}$. 


\section{Implementation of SVD filter for realistic geometries}

SVD filter requires the calculation of $\boldsymbol{B}=\boldsymbol{V} \boldsymbol{D}^{*} \boldsymbol{V}^{\boldsymbol{T}}$ matrix, which is of a size Nvoxel $\times$ Nvoxel, so impossible to store directly for full system. On the other hand, this matrix is diagonally dominant not only in the case of the 1D test system but for a 3D full system as well. Consequently it is enough to store the main diagonal and some side-diagonals. Thus, the remaining task is the calculation of the filtering matrix which requires a numerical SVD. As the calculation is still computationally tedious we present an alternative way. The simplified back projection accounts only for the qualities of the imaging system and the material dependent effects in the reconstructed volume are neglected. For the reconstruction, the activity of the voxels is assigned in a finite length vector. A forward projection-back projection operator composition (in matrix terms $\left.\boldsymbol{A}^{T} \boldsymbol{A}\right)$ maps a voxel space vector into another voxel space vector performing a low-pass filtering. In other words, a space-limited signal is low-pass filtered and space-limited again. The eigenfunctions of such an operator are the well-known prolate spheroidal wave functions (PSWF) ${ }^{21}$ and per definition these are the voxel space singular functions of the forward projection (and also the back projection) operator for simplified modelling case.

Observing (the square of) the singular value spectra of the system matrix, low-pass filtering is not ideal but PSWFs are good approximation for the singular vectors. Owing to this favourable property, matrix B can be calculated if singular value spectrum has been obtained (e.g. by means of inverse iteration). However, for an increased precision, certain generalisation of the functions is needed, as the low-pass filter (characterised by the squares of the singular values) is not ideal. This is done by using special spectral techniques from the theory of Sturm-Liouville operators applied to the Jacobi perturbed differential operator of Karoui et al. ${ }^{22}$

$\alpha=\beta=0$ case in Karoui et al..$^{22}$ corresponds to regular PSWFs, but $\alpha=\beta>0$ type generalisation gives a very good approximation for singular value spectrum and the voxel space singular vectors as well.

\section{Discussion}

In our paper all of the three SVD matrices from the factorisation of the system matrix were analysed. The results explained the perceived artefact as the convergence speed of the scheme with positron range modelling back projection was material dependent and the further advantage of the simplified back projection in terms of overall convergence speed and stability to noise. The presented SVD filter further amplified these favourable properties so as to fasten the algorithm but preserving its robustness. Additionally the use of faithful modelling was pointed out when high computational capacity is available.

A posteriori filtering is in close relation with such a priori methods when a regularizing term is added to the likelihood function in the problem formulation for decreasing noise sensitivity and accelerate the convergence. The resulted filtering term is then present usually in the nominator of the backprojection in additive form and arises from known constraints about the imaging process. In general, some kind of regularisation is almost always required due to the ill-posed nature of the reconstruction problem. Our SVD filter approaches from a bit different point of view: it does not require any a priori knowledge. The effect of $B$ matrix is not strictly regularisation but rather deconvolution which accelerates the convergence and the deconvolution process itself has to be regularised due to the presence of noise. Thus, the process has a filtering effect as well.

Monte Carlo simulations result slightly different system matrix elements through iterations which imply slightly different SVDs and B matrices. So as to make the most of the presented SVD filtering technique our further aim is to find the connection with conventional deconvolution methods which obtain exactly the same effect but the filtering factors can be recalculated in each iteration tailored to the given back projection system matrix. The special form (PSWF which is strongly connected to Fourier-transform ${ }^{22,23}$ ) of the singular vectors makes this direction promising.

\section{References}

1. Vardi Y, Shepp LA, Kaufman L. A statistical model for positron emission tomography (with discussion). J Am Stat Assoc 1985; 80: 8-20. doi: 10.2307/2288037.1981.389

2. McLachlan G, Krishnan T. The EM algorithm and extensions. New York: John Wiley and Sons, Inc; 1997.

3. Zeng GL. Medical image reconstruction. Heidelberg: Springer; 2010.

4. Szirmay-Kalos L, Magdics M, Toth B, Bukki T. Averaging and metropolis iterations for positron emission tomography. IEEE Trans Med Imaging 2013; 32: 589-600. doi: 10.1109/TML.2012.2231693

5. Zeng GL, Gullberg GT. Unmatched projector/backprojector pairs inan iterative reconstruction algorithm. IEEE Trans Med Imaging 2000; 19: 548-55. doi: $10.1109 / 42.870265$ 
6. Xin F, Hai-Peng W, Ming-Kai Y, Xiao-Li S, Xue-Xiang C, Shuang-Quan L, et al. PET image reconstruction with a system matrix containing point spread function derived from single photon incidence response. Chinese Phys $B$ 2015; 24: 018702. doi: 10.1088/1674-1056/24/1/018702

7. Gong K, Cherry SR, Qi J. On the assessment of spatial resolution of PET systems with iterative image reconstruction. Phys Med Biol 2016; 61: 193-202. doi: 10.1088/0031-9155/61/5/N193

8. Chávez-Rivera LB, Ortega-Máynez L, Mejía J, Mederos B. ML-EM reconstruction model including total variation for low dose PET high resolution data. Nuclear Science Symposium and Medical Imaging Conference (NSS/MIC), 2015 IEEE. doi: 10.1109/NSSMIC.2015.7582221

9. Gu XY, Zhou W, Li L, Wei L, Yin PF, Shang LM, et al. High resolution image reconstruction method for a double-plane PET system with changeable spacing. Chinese Phys C 2016; 40: 058201. doi: 10.1088/1674 1137/40/5/058201

10. Cserkaszky A, Légrády D, Wirth A, Bükki T, Patay G. GPU based Monte Carlo for PET image reconstruction: Parameter optimization. International Conference on Mathematics and Computational Methods Applied to Nuclear Science and Engineering (M\&C 2011), Rio de Janeiro, RJ, Brazil, May 8-12, 2011.

11. PANNI - PET aimed novel nuclear imager; forward Monte Carlo based image reconstruction software for positron emission tomography corresponding to code revision 664; General description; Coding: Cserkaszky A, Szlávecz Á, Légrády D, Tolnai G. Contact: legrady@reak.bme.hu

12. Wirth $A$, Cserkaszky $A$, Kéri $B$, Légrády $D$, Fehér $S$, Czifrus $S$, et al. Implementation of 3D Monte Carlo PET reconstruction algorithm on GPU. IEEE Nuclear Science Symposium Conference Records 2009; doi: 10.1109/ NSSMIC.2009.5402363.

13. Mediso Ltd. Product page of nanoScan. [citated 15 Jul 2017]. Available at http://www.mediso.hu/products. php?fid=2,11\&pid=90

14. Derenzo SE. Mathematical removal of positron range blurring in high resolution tomography. IEEE Nucl Sci 1986; 33: 565-9. doi: 10.1109/ TNS.1986.4337166

15. Lehnert W, Gregoire MC, Reilhac A, Meikle SR. Analytical positron range modelling in heterogeneous media for PET Monte Carlo simulation. Phys Med Biol 2011; 57: 4075-6. doi: 10.1088/0031-9155/56/11/009

16. Briesmeister JF. MCNP - a General Monte Carlo N-Particle Transport Code. Los Alamos National Laboratory Report LA-13709-M. April 2007

17. Liu Z, Obi T, Yamaguchi M, Ohyama N. An investigation of convergence rates in expectation maximization (EM) iterative reconstruction. IEEE Nuclear Science Symposium Conference Record 1999. doi: 10.1109/ NSSMIC.1999.842824

18. Hansen PC. Numerical tools for analysis and solution of Fredholm integral equations of the first kind. Inverse Problems 1992; 8: 849-72. doi: https:// doi.org/10.1088/0266-5611/8/6/005

19. Magdics M, Szirmay-Kalos L, Toth B, Penzov A. Analysis and control of the accuracy and convergence of the ML-EM iteration. Lecture notes in computer science. Berlin: Springer; 2014. p. 147-54.

20. Pyzara A, Bylina B, Bylina J. The influence of a matrix condition number on iterative methods convergence. IEEE Proceedings of the Federal Conference on Computer Science and Information Systems; 2011. p. 459-64.

21. Prolate spheroidal wave functions. Wikipedia. [cited $15 \mathrm{Jul}$ 2017]. Available at https://en.wikipedia.org/wiki/Prolate_spheroidal_wave_function

22. Karoui A, Souabni A. Generalized prolate spheroidal wave functions: Spectral analysis and approximation of almost band-limited functions. J Fourier Anal Appl 2015; 22: 382-412. doi: 10.1007/s00041-015-9420-3

23. Moore IC, Cada M. Prolate spheroidal wave functions, an introduction to the Slepian series and its properties. Appl Comput Harmon Anal 2004; 16 208-230. doi: 10.1016/j.acha.2004.03.004 